\title{
Comments on the perturbative and non-perturbative contributions in unpolarized SIDIS
}

\author{
J. Osvaldo Gonzalez-Hernandez* \\ Author University of Turin \\ E-mail: joseosvaldo.gonzalez@to.infn.it
}

We address the large suppression of the unpolarized SIDIS cross section in the small transverse momentum region, due to the $O\left(\alpha_{\mathrm{s}}\right)$ contributions in the $C$-coefficients needed for a consistent matching to the large transverse momentum region, at this order in the CSS factorization scheme. We will show that a TMD analysis with the type of cuts as those in recent extractions cannot describe the data at this order.

XXVII International Workshop on Deep-Inelastic Scattering and Related Subjects - DIS2019 8-12 April, 2019

Torino, Italy

${ }^{*}$ Speaker. 
An analysis of multidimensional unpolarized SIDIS data, with maximal perturbative input, is still missing in the literature. Here, by maximal perturbative input we mean the kind of picture implemented in [1] for $W$ and $Z$ production, where soft gluon effects of the type arising in a TMDlike formalism are accounted for, as well as the interplay and matching with the large transverse momentum region. For instance, analyses like that of [2] do not account for soft gluon effects, thus lacking TMD-evolution. This leading order formalism with a small number of parameters, has been shown to be a reasonable picture when the ranges of the hard scale do not vary largely, but does not allow to extrapolate results to higher energies. More recently, in a simultaneous analysis of unpolarized SIDIS, Drell-Yan and $Z$ production [3], a large amount of data was described, including soft gluon effects, in the language of resumation, up to next-to-leading log (NLL), although lacking the beyond-leading-order corrections necessary to match to the large transverse momentum region. Furthermore, this analysis required an ad-hoc treatment in the normalization of the cross section in order to describe the SIDIS data.

Including maximal perturbative input should further constrain the extraction of TMDs providiong more predictive power ${ }^{1}$. There are, however, several challenges in the way. For instance, the difficulty in smoothly matching the low and large transverse momentum regions, and the undershooting of theoretical calculations w.r.t data, employing modern collinear functions sets in the large transverse momentum regime in different processes [4, 5, 6, 7]. Some progress has been made in these regards, and it is likely that in the near future such issues may be better understood (see for instance $[8,9,10])$.

In this work we address one more complication, which has not yet been discussed in detail in the literature: the order $\alpha_{\mathrm{s}}$ contributions in the TMDs, needed for a consistent matching at this order, largely supress the cross-section in the small transverse momentum region. In fact, as we will show in the following examples, an analysis with the type of cuts as those used in [2,3] cannot describe the data at this order. For concreteness, we work within the so called CSS1 formalism [11]. The results presented here should not depend on the particular factorization scheme employed, see [12] for a detailed comparison of different frameworks. In fact, using CSS1 allows for a more direct comparison to the available analyses of unpolarized SIDIS data.

In CSS1, the unpolarized SIDIS cross section reads

$$
\begin{aligned}
\frac{d \sigma^{\text {total }}}{d x d y d z d q_{\mathrm{T}}^{2}} & =\pi \sigma_{0}^{\mathrm{DIS}} \int \frac{d^{2} b_{\mathrm{T}} e^{\mathrm{i} q_{\mathrm{T}} \cdot b_{\mathrm{T}}}}{(2 \pi)^{2}} W^{\mathrm{SIDIS}}\left(x, z, b_{\mathrm{T}}, Q\right)+Y^{\mathrm{SIDIS}}\left(x, z, q_{\mathrm{T}}, Q\right), \\
W^{\mathrm{SIIS}}\left(x, z, b_{\mathrm{T}}, Q\right) & =\sum_{j} e_{j}^{2} \sum_{i, k}\left(C_{j i}^{\mathrm{in}} \otimes f_{i}\left(x, \mu_{\mathrm{b}}^{2}\right)\right)\left(C_{k j}^{\text {out }} \otimes D_{k}\left(z, \mu_{\mathrm{b}}^{2}\right)\right) \exp \left[S\left(b_{\mathrm{T}}, x, z, Q\right)\right], \\
S\left(b_{\mathrm{T}}, x, z, Q\right) & =-\int_{\mu_{\mathrm{b}}^{2}}^{Q^{2}} \frac{d \mu^{2}}{\mu^{2}}\left[A\left(\alpha_{\mathrm{s}}(\mu)\right) \ln \left(\frac{Q^{2}}{\mu^{2}}\right)+B\left(\alpha_{\mathrm{s}}(\mu)\right)\right]+S_{\text {model }}\left(x, z, b_{\mathrm{T}}, Q\right) .
\end{aligned}
$$

For details on the structure of Eqs. (1-3), see for instance [13]. For the purposes of our dicussion, we remark only a few points:

1. The CSS1 expression for the cross section should describe observables up to a range of $q_{\mathrm{T}} \sim Q$, provided the corrections of the $Y^{\mathrm{SIDIS}}$ are included.

\footnotetext{
${ }^{1}$ It is likely that such improvement will be reflected in a reduction of the number of parameters necessary to describe data
} 
2. $A, B, C_{j i}^{\text {in }}$ and $C_{k j}^{\text {out }}$ are calculable within pQCD, while $S_{\text {model }}$ should be extracted from data.

3. The convolutions of the matching coefficients $C^{\text {in/out }}$ with collinear distributions and fragmentation functions, $f_{i / A}\left(x, \mu_{\mathrm{b}}^{2}\right)$ and $D_{B / k}\left(z, \mu_{\mathrm{b}}^{2}\right)$, provide an important constraint in any TMD formalism. The scale $\mu_{\mathrm{b}}=2 e^{-\gamma_{E}} / b_{*}$ involves a "freezing" prescription, which most often is taken to be

$$
b_{*}=\frac{b_{\mathrm{T}}}{\sqrt{1+b_{\mathrm{T}}^{2} / b_{\max }^{2}}} .
$$

Since we are not attempting a full extraction, we will focus only on the COMPASS 2017 data [14]. Although not explicitly shown here, similar issues arise for the other available multidimensional sets.

We wish to show the suppression of the cross section in the small transverse momentum region, due to $O\left(\alpha_{s}\right)$ corrections. We will illustrate this by performing test fits on data at fixed $x$ and $Q^{2}$, that is, we fit individual panels in Fig. 1. Thus, for each example we perform 22 independent fits. These test fits are useful in a full analysis because they allow us to proceed stepwise: first testing our ability to reproduce one panel at a time, taking a first glance at the $z$ and $P_{\mathrm{T}}$ dependence, and then extending the model so that also $x$ and $Q^{2}$ dependences can be included. In fact, the first step can be seen as a necessary condition to perform a simultanous fit on the entire data set. Our examples will show that at $O\left(\alpha_{\mathrm{s}}^{1}\right)$, such condition cannot be fullfilled when considering the type of cuts employed in [2,3]. More concretely, we trace this suppression back to the $O\left(\alpha_{s}\right)$-terms in the matching coefficients $C_{j i}^{\text {in }}$ and $C_{k j}^{\text {out }}$. We quantify this by introducing a normalization parameter $N_{i}$, for each test fit, which will serve as a diagnostics tool to quantify the severity of the problem. We proceed as follows:

1. We consider only the contributions from $W^{\mathrm{SIDIS}}$ in Eq. (1), with $S_{\text {model }}=-g_{1} b_{\mathrm{T}}^{2}-g_{2} b_{\mathrm{T}}^{2} / z^{2}$, where $g_{1}$ and $g_{2}$ are free parameters ${ }^{2}$. Furthermore, $b_{\max }=1.0 \mathrm{GeV}^{-1}$ in Eq. (4).

2. Test fits are performed on the COMPASS 2017 data [14], for fixed $x$ and $Q^{2}$ (22 fits in total). A normalization $N_{i}$ is introduced for each test fit, multiplying Eq. (1) (22 normalizations in total). This free parameters will serve as a diagnostics tool to quantify the severity of the problem, the suppression of the cross section in the small transverse mometum region. In an ideal scenario, all the normalizations should satisfy $N_{i}=1$.

3. The test fits are performed on COMPASS 2017 data in the region $P_{\mathrm{T}} \leq 0.9 \mathrm{GeV}^{3}$. Note that the reported data are actually multiplicities: SIDIS cross sections normalized by fully inclusive cross sections.

4. Although not explictly shown here, in the cases where large values for $N_{i}$ are encountered, we check that performing a fit with fixed $N_{i}=1$ does in fact result in undershooting the data.

\footnotetext{
${ }^{2}$ The issue we want to discuss at small transverse momentum is not expected to be resolved by means of the $Y$-term, which corrects the cross section in the region of intermediate $q_{\mathrm{T}}$.

${ }^{3}$ Strictly following the CSS formalism one should perform cuts in $q_{\mathrm{T}}$, however our choice allows for a more direct comparison with current analyses on COMPASS data. We have checked that cutting the data for $q_{\mathrm{T}}<0.5 Q$ renders similar results.
} 
We remark some important aspects of our procedure. First, the values of $N_{i}$, used to quantify the issues with normalization in the fits, do not need to be exactly unity to correspond to a satisfactory picture. For instance, one can tolerate a small deviation $N_{i} \pm \delta_{i}$, provided $\delta_{i}$ is consistent in all or in a given set of test fits. In this situation one may expect to fit simultaneously the corresponding range of kinematics by suplementing the calculation with one unique normalization, as tipically done in global analyses. Second, in some cases the additional flexibility due to $N_{i}$ may not be required to fit the data. This can be reflected in large statistical uncertainties or strong correlations between the model parameters and $N_{i}$. Finally, details of the implementation do have an impact in the precise minimal values of the $N_{i}$, so it is possible that improvements can be made by adjusting, for instance, the model or the value of $b_{\max }$.

With this considerations in mind, we will focus on the situations where large deviations from unity are observed for $N_{i}$. We proceed by performing test fits for three different cases, summarized in Table 1.

\begin{tabular}{|c|ccc|}
\hline & $A$ & $B$ & $C^{\text {in/out }}$ \\
\hline CASE I & $O\left(\alpha_{\mathrm{s}}^{0}\right)$ & $O\left(\alpha_{\mathrm{s}}^{0}\right)$ & $O\left(\alpha_{\mathrm{s}}^{0}\right)$ \\
\hline CASE II & $O\left(\alpha_{\mathrm{s}}^{2}\right)$ & $O\left(\alpha_{\mathrm{s}}^{1}\right)$ & $O\left(\alpha_{\mathrm{s}}^{0}\right)$ \\
\hline CASE III & $O\left(\alpha_{\mathrm{s}}^{2}\right)$ & $O\left(\alpha_{\mathrm{s}}^{1}\right)$ & $O\left(\alpha_{\mathrm{s}}^{1}\right)$ \\
\hline
\end{tabular}

Table 1: Cases considered in our test fits. The columns show the order to which each of the perturbative coefficients in Eqs. (1-3) are considered.

The first case is a leading order picture, not very different from the generalized parton model of [2], within the scope of this work. The second one corresponds to the same accuracy in perturbation theory of the analysis in [3]. There, this is called LO/NLL. In this case, the matching coefficients $C^{\text {in/out }}$ are unity, thus the convolutions in Eq. (2) become simply $f_{\mathrm{j}}\left(x, \mu_{\mathrm{b}}\right)$ and $D_{\mathrm{j}}\left(z, \mu_{\mathrm{b}}\right)$. Finally, the third case is similar to the second one but now including corrections of $O\left(\alpha_{\mathrm{s}}^{1}\right)$ in the matching coefficients $C^{\text {in/out }}$, thus the convolutions in Eq. (2) are non-trivial. These corrections are necessary to match the small and large transverse momentum regions in the CSS formalism, thus a crucial step in a TMD analysis.

We note that for our discussion, it is more important to look at each one of these cases in comparison with the other two, rather than reading out precise values of the extracted parameters. We simply aim at exemplifying the most prominent issue in describing the data in the small transverse momentum region.

Results for the test fits described in Table 1 are shown in Fig. 1. There, the obtained values of $N_{i}$ are represented by color bars on top of each panel. For instance, in both cases I and II, in the the top right panel (labeled as "22"), the test fits result in good agreement with the data, with values for the normalization $N_{22} \sim 1$. However, in this same panel in case III, a description of the data can only be achieved with a value for the normalization $N_{22} \sim 2$. In fact, for most of the kinematics in case III, the suplemental normalizations $N_{i}$ are mostly close to this same value. This means that the actual computation of the cross section in case III is supressed by about roughly $50 \%$ in most panels w.r.t. cases I and II. By comparing the three plots in Fig. 1, it is clear that such suppression arises from the $O\left(\alpha_{\mathrm{s}}^{1}\right)$ contributions of the matching coefficients $C^{\text {in/out }}$. It is also interesting to 
note, by comparing cases I and II in Fig. 1, how the situation improves for some of the panels in the lower $x$ region, when considering soft gluon effects, i.e. when $A$ and $B$ in Eq. (3) are non-trivial.

We should stress that the suplemental normalizations $N_{i}$ in our test fits have been used as a diagnostics tool only. We do not attempt to justify their use in a full TMD analysis. On the contrary, it must be emphasized that there is a the danger in doing so: this type of treatment for the normalizations, in a TMD extraction, may render the constraints provided by the matching coefficients useless, thus undermining the predictive power of pQCD. This is so because the role of such normalization is to enhance(supress) the contributions from $C^{\text {in/out }}$ in the small $b_{\mathrm{T}}$ region, but the small $b_{\mathrm{T}}$ behaviour of $W^{\text {SIDIS }}$ in Eq. (2), should be determined solely by the convolutions invoving the coefficients $C^{\text {in/out }}$.

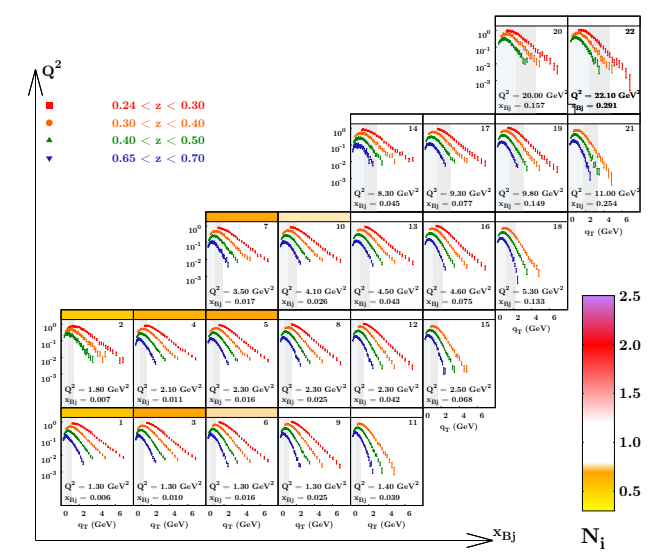

Case I

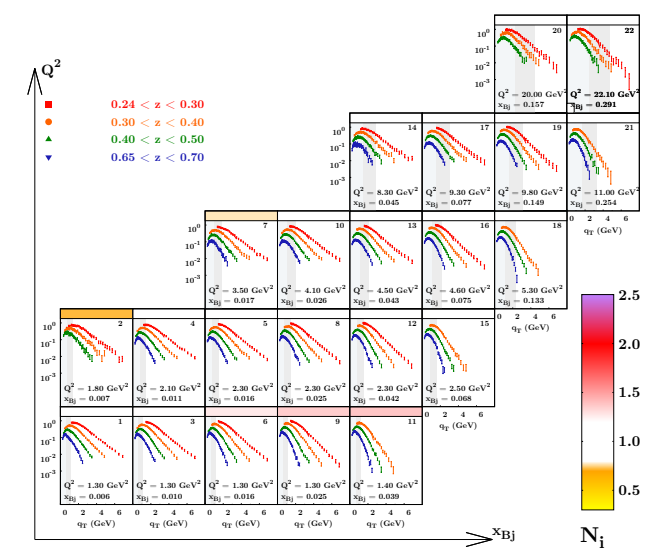

Case II

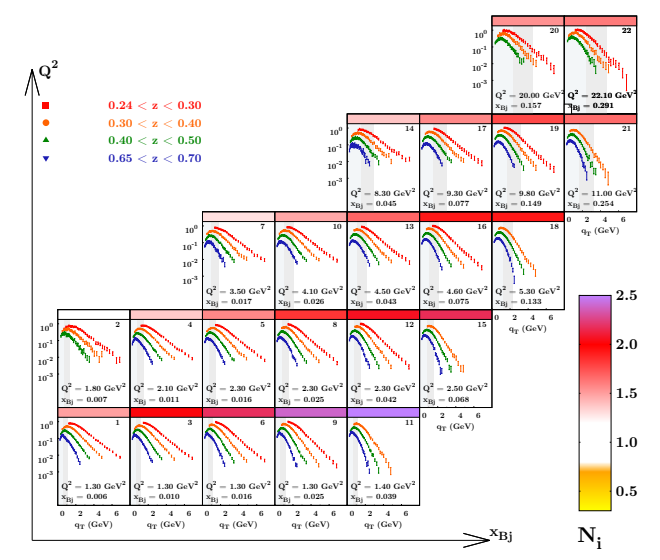

Case III

Figure 1: Test fits for cases I, II \& III in Table 1. In each case, fits are performed panel by panel, further details in text.

The suppression of the SIDIS cross section due to the $O\left(\alpha_{\mathrm{s}}^{1}\right)$ contributions of the matching coefficients $C^{\text {in/out}}$, poses a challenge for TMD studies. We stress that while we analysed data in the region $P_{\mathrm{T}}<0.9 \mathrm{GeV}$, we have checked that selecting data in the region $q_{\mathrm{T}}<0.5 Q$, which is more consistent with the CSS formalism, renders similar results. It is possible that this issue reflects the fact that a more stringent cut should be imposed. In fact, the analysis in [16] points to a 
cut of $q_{\mathrm{T}}<0.2 Q$, for the TMD region. Finally, we remark that the choice of model does not seem to resolve the issue. We found that with a power law behaviour for $\exp \left(S_{\text {model }}\right)$, some improvement is attained, but not enough to resolve the issue. We leave a more detailed discussion on these topics for an upcoming publication.

\section{References}

[1] P. B. Arnold and R. P. Kauffman, $W$ and $Z$ production at next-to-leading order: From large $q_{T}$ to small, Nucl. Phys. B349 (1991) 381.

[2] M. Anselmino, M. Boglione, J. O. Gonzalez Hernandez, S. Melis and A. Prokudin, Unpolarised Transverse Momentum Dependent Distribution and Fragmentation Functions from SIDIS Multiplicities, JHEP 04 (2014) 005 [1312.6261].

[3] A. Bacchetta, F. Delcarro, C. Pisano, M. Radici and A. Signori, Extraction of partonic transverse momentum distributions from semi-inclusive deep-inelastic scattering, Drell-Yan and Z-boson production, 1703.10157.

[4] M. Boglione, J. O. G. Hernandez, S. Melis and A. Prokudin, A study on the interplay between perturbative QCD and CSS/TMD formalism in SIDIS processes, JHEP 02 (2015) 095 [1412.1383].

[5] J. O. Gonzalez-Hernandez, T. C. Rogers, N. Sato and B. Wang, Challenges with Large Transverse Momentum in Semi-Inclusive Deeply Inelastic Scattering, Phys. Rev. D98 (2018) 114005 [1808.04396].

[6] A. Bacchetta, G. Bozzi, M. Lambertsen, F. Piacenza, J. Steiglechner and W. Vogelsang, Difficulties in the description of Drell-Yan processes at moderate invariant mass and high transverse momentum, 1901.06916.

[7] E. Moffat, T. C. Rogers, N. Sato and A. Signori, Collinear Factorization in Wide-Angle Hadron Pair Production in $e^{+} e^{-}$Annihilation, 1909.02951.

[8] J. Collins, L. Gamberg, A. Prokudin, T. C. Rogers, N. Sato and B. Wang, Combining TMD factorization and collinear factorization, 2017, 1702.00387, https://inspirehep.net/record/1511695/files/arXiv:1702.00387.pdf.

[9] M. G. Echevarria, T. Kasemets, J.-P. Lansberg, C. Pisano and A. Signori, Matching factorization theorems with an inverse-error weighting, Phys. Lett. B781 (2018) 161 [1801.01480].

[10] M. Boglione, A. Dotson, L. Gamberg, S. Gordon, J. O. Gonzalez-Hernandez, A. Prokudin et al., Mapping the Kinematical Regimes of Semi-Inclusive Deep Inelastic Scattering, Submitted to: J. High Energy Phys. (2019) [1904.12882].

[11] J. C. Collins, D. E. Soper and G. Sterman, Transverse momentum distribution in Drell-Yan pair and $W$ and Z boson production, Nucl. Phys. B250 (1985) 199.

[12] J. Collins and T. C. Rogers, Connecting Different TMD Factorization Formalisms in QCD, 1705.07167.

[13] P. Nadolsky, D. R. Stump and C. P. Yuan, Semi-inclusive hadron production at HERA: The effect of QCD gluon resummation, Phys. Rev. D61 (1999) 014003 [hep-ph/9906280].

[14] COMPASS collaboration, Transverse-momentum-dependent Multiplicities of Charged Hadrons in Muon-Deuteron Deep Inelastic Scattering, Phys. Rev. D97 (2018) 032006 [1709. 07374 ]. 
[15] COMPASS collaboration, Hadron transverse momentum distributions in muon deep inelastic scattering at 160 gev/c, Eur. Phys. J. C73 (2013) 2531 [1305. 7317].

[16] I. Scimemi and A. Vladimirov, Analysis of vector boson production within TMD factorization, Eur. Phys. J. C78 (2018) 89 [1706.01473]. 